\section{REMARKS ON THE RELATION OF THE UTERUS TO DESCENT OF THE PELVIC FLOOR, AND TO GEN. \\ ITAL PROLAPSE.}

Presented to the Section on Obstetrics and Jiseases of Women at the Forty-eighth Annlial Meeting of the American Medical Association, held at Philadelphia, P'a., June 1-4, 1897.

BY GEO. ERETY SHOEMAKER, M.D. GYNECOLOGIST TO METHODIST HOSPITAI. P'HILADEITIIIA.

As the title implies, it is not intended here to formally cover the field of genital prolapse but to remark upon one phase of it, and to emphasize the fact that the uterus is not the sole offender, but that, in greater or less degree, all the other pelvic organs and tissues are involved to such an extent in some cases that even after the uterus is completely restored to its position, the prolapse of vagina, rectum, bladder and tissues of the pelvic floor continues.

There seems to be, in the minds of some, an idea that the uterus descends alone through a normal tube, the vagina, because of some relaxation of suspending supports above. This idea leads to attempts to cure prolapse by shortening round ligaments alone, by fixation (literal) of the uterus alone to the abdominal wall, or even to the performance of hysterectomy followed by fastening the cervical stump to higher pelvic tissues, while the relaxation, sliding and stretching which have occurred in the pelvic diaphragm are left untreated. Ultimate failure to cure must be the result of this policy. The truth is that in varying degree in different cases, the uterine prolapse is only a part of a vast hernia through the inferior pelvic strait of the pelvis; that in this hernia are involved bladder, rectum, peritoneal pouches in front and behind the uterus, with or without bowel contents; also the muscles and fasciæe of the pelvic floor. All of these structures do not descend to equal degree, but there is a gradual sliding in different planes. The uterus is often forced furthest down by intra-abdominal pressure and gravity, the posterior bladder wall less so, the anterior bladder wall still less so, and so on with each succeeding plane of connective tissue until the bony pelvis is reached. By thrusting up the uterus, this whole prolapsed mass may be made to telescope back, partly into position, but not wholly. No operation can be permanently successful which aims to deal with the u terus alone and does not take into account the sliding planes of stretched tissue and the telescoping movements when the cervix is thrust up or down.

This is best illustrated by the following case, which shows that the uterus may be even higher than its normal place in the pelvis and yet the bladder and much of the vagina be outside the vulva.

Mrs. X., a young and healthy multipara, was referred to me for operation by her physician because her "womb came out," giving rise to chafing and various discomforts. He had made no careful examination, but saw simply a large pinkish mass, the size of the fist, protruding from the vulva, and sent her to me without further investigation. Upon examination I found, indeed, a large genital prolapse outside the vulva, larger than the fist, but it was made up of anterior vaginal wall and bladder in front, and rectocele behind. The uterus so far from being an element in the case was, to the surprise of patient and physician, found to be pregnant six months. It was within the abdominal cavity, having risen normally above the supe- rior strait. The cervix was normal in size and high up. Here was a case in which Nature by pregnancy had restored the uterus temporarily to its normal positionin the body without dragging up enough of the other elements of the genital hernia to attract the patient's attention. In fact, the prolapse continued. A hundred diagrams or theories could not better demonstrate the position that any effort to cure this woman by any operation for suspending or removing the uterus would be futile unless something were also done to the pelvic floor. The case aborted shortly afterward and is now awaiting operation. What shall this be? First a serious attempt from below to restore, not the perineum but the integrity of the pelvic floor, of the whole pelvic diaphragm. By operating within the vagina, to endeavor to reach with stitches and take up the higher and deeper planes of pelvic fascia and muscle. When the uterus is large and the cervix long or hypertrophied, amputation of the cervix from below is a great help as it is preceded by dissecting off the vagina from the cervix, while the method of sewing shoriens materially the vagina and implants it higher upon the uterus, virtually at the same time taking a reef in utero-sacral attachments. In addition I believe that uterine suspesion should usually be done, or in very rare cases of enlarged uterus, hysterectomy, but the main effort must be concentrated upon the vagina.

It is of interest, in passing, to note the curious tolerance of this patient to septic influences, no doubt owing to long protrusion of the uterus from the body. She aborted, as has been said, at six months. Her physician found, a few days later, to his horror, that finding herself unable to pass her urine owing to protrusion of the bladder she would take her hands and push everything within the vulva, when the husband with unwashed hands would catheterize her with an instrument wnich he had bought for that purpose. No harm resulted!

\section{THE INFLUENCE OF OUR SCHOOL SYSTEM ON THE HEALTH AND DEVELOP. MENT OF THE CHILD.}

resented to the Section on Diseases of Children, at the Forty-eighth Annual Meeting of the American Medical Association, beld at Philadelphia, Pa., June 1-4, 1897.

BY E. STUVER, M.Sc., M.D., PH.D.

Member Colorado State Medical Society (Vice-president 1894), American Medical Association, International Medical Congress (Berlin); Active Member National Educational Association. RAWLINS, WYO.

(Concluded from page 1252.)

Answers to No. 6.-To most persons it may seem that such a question as this is entirely superfluous; that the dictates of humanity and common sense would make such a practice an impossibility. From my personal experience as a member of a school board and as a practicing physician however, I am sorry to say that the practice is more prevalent than I would have believed it to be without a thorough investigation of the subject. Indeed, I am satisfied that even superintendents and principals of schools do not fully appreciate how frequently teachers err in this matter. This can probably be explained, in part at least, by the fact that it is a delicate subject, about which parents do not like to make complaint, and which does not receive the attention and discussion in teachers' meetings that its importance demands. Whatever may be the cause of the practice it certainly exists to a 